\title{
WIIBRAGEC

\section{BOAS PRÁTICAS NA REDUÇÃO, REUTILIZAÇÃO E RECICLAGEM DE RESÍDUOS NA CONSTRUÇÃO DE EDIFÍCIOS}

\author{
QUEIROZ, Maria Ezir Rodrigues de; OLIVEIRA, Jaqueline Mata de; \\ LORDSLEEM JÚNIOR, Alberto Casado
}

(1) Universidade de Pernambuco, ezir-queiroz@hotmail.com, (2) Universidade de Pernambuco, jmo@poli.br, (3) Universidade de Pernambuco, acasado@poli.br

\begin{abstract}
RESUMO
A indústria da construção civil é uma das principais responsáveis pela grande geração de resíduos. Os resíduos da construção civil (RCC), comumente chamados de entulhos, provocam grandes impactos ambientais quando não são geridos e destinados adequadamente. Por isso, faz-se necessária a aplicação de práticas que visem reduzir, reutilizar e reciclar (3Rs) os resíduos gerados pela construção civil no canteiro de obras. Este trabalho tem como objetivo apresentar práticas de redução, reutilização $e$ reciclagem dos RCC. A metodologia de pesquisa consistiu em uma revisáo bibliográfica utilizando a metodologia de revisão sistemática PRISMA (Preferred Reporting Items for Systematic reviews and MetaAnalyses) através do Portal de Periódicos da CAPES (Coordenação de Aperfeiçoamento de Pessoal de Nivel Superior) e de outras bases, como Google Scholar e ScienceDirect. Em seguida, realizou-se uma pesquisa de campo em uma obra localizada na cidade do Recife, Estado de Pernambuco, onde foi aplicado um questionário específico para análise da gestão de resíduos na obra. Os resultados obtidos demonstram diversas ações de redução, reutilização e reciclagem realizadas em canteiros de obras. A principal contribuição deste trabalho consiste em identificar e descrever boas práticas para uma melhor gestão dos resíduos da construção civil quanto aos $3 R$ s.
\end{abstract}

Palavras-chave: Resíduos, Redução, Reutilização, Reciclagem, Construção Civil.

\begin{abstract}
The construction industry is one of the main responsible for the large generation of waste. Construction waste (RCC), commonly called rubble, causes major environmental impacts when not properly managed and disposed of. Therefore, it is necessary to apply practices that aim to reduce, reuse and recycle (3Rs) the waste generated by civil construction at the construction site. This work aims to present practices for the reduction, reuse and recycling of RCC. The research methodology consisted of a bibliographic review using the systematic review methodology PRISMA (Preferred Reporting Items for Systematic reviews and Meta-Analyzes) through the CAPES (Coordenação de Aperfeiçoamento de Pessoal de Nivel Superior) journals and other databases, like Google Scholar and ScienceDirect. Then, a field research was carried out in a work located in the city of Recife, State of Pernambuco, where a specific questionnaire was applied to analyze the waste management in the work. The results obtained demonstrate several reduction, reuse and recycling actions carried out in construction sites. The main contribution of this work is to identify and describe good practices for better management of construction waste in relation to the $3 R$ s.
\end{abstract}

Keywords: Waste, Reduction, Reuse, Recycling, Construction.

\footnotetext{
1 QUEIROZ, M. E. R. de; OLIVEIRA, J. M. de; LORDSLEEM JÚNIOR, A. C. Boas práticas na redução, reutilização e reciclagem de resíduos na construção de edifícios. In: SIMPÓSIO BRASILEIRO DE GESTÃO E ECONOMIA DA CONSTRUÇÃO, 12., 2021, Maceió. Anais[...] Porto Alegre: ANTAC, 2021. p.1-8. Disponível em: https://eventos.antac.org.br/index.php/sibragec/article/view/436. Acesso em: 2 out. 2021
} 


\section{INTRODUÇÃO}

No Brasil, o aumento da densidade demográfica e o acelerado processo de urbanização são os principais fatores responsáveis pelo aumento da demanda por edificações. Em contrapartida, a indústria da construção civil se destaca pelo alto consumo de recursos naturais e pela grande geração de resíduos.

Apesar de ser economicamente fundamental para o país, a indústria da construção civil é responsável pela utilização de até $50 \%$ do total de recursos naturais consumidos pela sociedade (MONTEIRO, AMARAL E DELGADO, 2017). Além disso, de acordo com Silva, Santos e Araújo (2017), a construção civil é responsável pela geração de até 70\% dos resíduos sólidos urbanos.

Com relação aos resíduos produzidos pela construção civil, a ABRECON (2017) apresentou em sua pesquisa setorial que $70 \%$ desses resíduos poderiam ser reutilizados no processo construtivo em obras. Visto isso, as práticas de redução, reutilização e reciclagem (3Rs) são alternativas eficazes no tratamento ambientalmente adequado dos resíduos da construção civil (RCC). Este artigo tem como objetivo sistematizar essas práticas.

\section{RESÍDUOS DA CONSTRUÇÃO CIVIL}

A geração de resíduos da construção civil no Brasil é regulamentada pela Resolução ${ }^{\circ}$ 307/2002 do CONAMA (Conselho Nacional do Meio Ambiente), a qual classifica os resíduos em 4 classes (A, B, C e D) estabelecendo o objetivo das práticas de redução, reutilização e reciclagem. Ainda segundo ela, prioritariamente as empresas construtoras devem ter como objetivo a não geração de resíduos e, em seguida, a redução, reutilização, reciclagem, tratamento e disposição final ambientalmente adequada dos resíduos da construção civil.

Como instrumento para operacionalizar a gestão de resíduos, o Plano de Gerenciamento de Resíduos da Construção Civil (PGRCC) é o documento técnico a ser elaborado para a obra com o objetivo de estabelecer um conjunto de procedimentos específicos para o atendimento da Resolução n ${ }^{\circ}$ 307/2002 do CONAMA. As cinco etapas que devem constar no PGRCC são: caracterização, segregação, acondicionamento, transporte e destinação.

De acordo com a pesquisa setorial realizada pela ABRECON (2017), 60\% do total de resíduos sólidos urbanos gerados no Brasil advém da construção civil. A ABRECON (2015) destacou ainda que grande parte dos municípios brasileiros ainda destinavam os RCC a locais irregulares e que apenas $20 \%$ dos RCC gerados eram reciclados.

Portanto, a indústria da construção civil é causadora de grande impacto negativo ao meio ambiente, tanto pelo elevado consumo de recursos naturais, quanto pela disposição irregular dos resíduos gerados. Os dados anteriores mostram a necessidade da aplicação de técnicas de redução, reutilização e reciclagem dos resíduos no canteiro de obras.

\section{METODOLOGIA}

A pesquisa exploratória objetiva proporcionar maior familiaridade com o problema tornando-o mais explícito e viabilizando a construção de hipóteses, como também aprimorar ideias e descobrir instituições. Esta classificação de pesquisa envolve levantamento bibliográfico, entrevistas com pessoas que possuem experiências práticas com o tema e a análise de exemplos que permitam uma melhor compreensão (GIL, 2010). A pesquisa deste trabalho é exploratória e a metodologia utilizada consistiu nas etapas que estão apresentadas no Quadro 1. 
Quadro 1 - Etapas da metodologia utilizada

\begin{tabular}{|l|l|}
\hline Etapas & Características \\
\hline Etapa 1 & $\begin{array}{l}\text { Realização da revisão bibliográfica sobre o tema desta pesquisa, utilizando a metodologia de } \\
\text { revisão sistemática PRISMA (Preferred Reporting Items for Systematic reviews and Meta- } \\
\text { Analyses) através do Portal de Periódicos da CAPES (Coordenação de Aperfeiçoamento de } \\
\text { Pessoal de Nível Superior) e também do Google Scholar e ScienceDirect. Além disso, também } \\
\text { foram realizadas buscas por trabalhos de conclusão de curso e por dissertações de mestrado } \\
\text { Sobre o tema desta pesquisa. }\end{array}$ \\
\hline Etapa 2 & $\begin{array}{l}\text { Lins (2020), através de sua pesquisa realizada na EMLURB (Autarquia de Manutenção e } \\
\text { Limpeza Urbana do Recife), obteve os indicadores de geração de resíduos de obras com suas } \\
\text { respectivas empresas na cidade do Recife, estado de Pernambuco. Os indicadores foram } \\
\text { selecionados de acordo com os seguintes critérios: os resíduos gerados na etapa de construção, } \\
\text { o uso da edificação como residencial e a tipologia de edificação como conjunto de } \\
\text { apartamentos. Em seguida, foi elaborado um ranking com as empresas construtoras que } \\
\text { obtiveram os menores indicadores de geração de resíduos, uma delas teve sua obra como alvo } \\
\text { da pesquisa de campo. }\end{array}$ \\
\hline Etapa 3 3 & $\begin{array}{l}\text { Elaboração do checklist com as informações necessárias para ser aplicado na pesquisa de } \\
\text { campo. Foram coletados a partir do checklist os seguintes itens: dados da empresa construtora, } \\
\text { do respondente e da obra; se há a realização da compatibilização de projetos; controle de } \\
\text { produção de materiais no canteiro de obras; logística reversa; elaboração, implantação e } \\
\text { monitoramento das 5 etapas do PGRCC; layout do canteiro; ações de não geração de resíduos } \\
\text { no canteiro de obras; ações de redução; ações de reutilização; ações de reciclagem. }\end{array}$ \\
\hline Etapa 4 & $\begin{array}{l}\text { Realização do estudo de caso em uma obra de edifício residencial na cidade do Recife, estado } \\
\text { de Pernambuco. Aplicação do checklist, buscando focar a investigação nas práticas de redução, } \\
\text { reutilização e reciclagem que foram realizadas na obra. Além disso, foram capturadas fotos } \\
\text { para complementar o estudo de caso. }\end{array}$ \\
\hline
\end{tabular}

Fonte: Autores

A metodologia Prisma, apresentada como a Etapa 1 no Quadro 1, obteve resultados importantes com relação às ações de redução, reutilização e reciclagem que devem ser frisados.

\subsection{Metodologia Prisma}

Os resultados da seleção das bibliografias nas bases de dados foram obtidos por meio de buscas a partir de palavras-chave relevantes sobre o tema e, em seguida, foram aplicados critérios de triagem e elegibilidade.

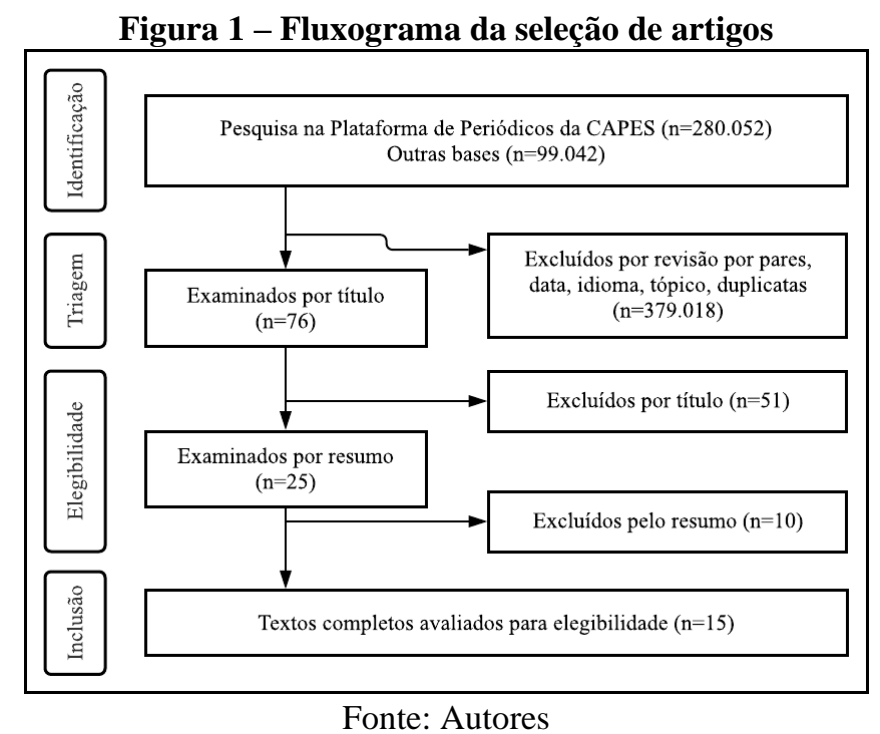

Portanto, após realizar a pesquisa no Portal de Periódicos da CAPES, no Google Scholar e no ScienceDirect e, em seguida, fazer a aplicação dos critérios conforme apresentado na Figura 1, o resultado total da pesquisa bibliográfica utilizando a metodologia PRISMA correspondeu a 15 bibliografias para análise completa. 
A partir da análise dos artigos, foi possível coletar diversas ações relacionadas aos 3Rs. Os Quadros 2, 3 e 4 apresentam, separadamente, as principais ações de redução, reutilização e reciclagem de resíduos, respectivamente.

Quadro 2 - Ações de Redução dos RCC

\begin{tabular}{|l|l|}
\hline Autor/Ano & Ações de Redução \\
\hline Gangolells et al. (2014) & $\begin{array}{l}\text { Armazenamento adequado dos insumos para evitar danos prematuros } \\
\text { causados por quebras e deterioração devido a umidade. }\end{array}$ \\
\hline Brasileiro e Matos (2015) & $\begin{array}{l}\text { Substituição de parte dos agregados tradicionais por agregados reciclados, } \\
\text { como substituição de agregado de concreto ou de mármore para produção de } \\
\text { concreto asfáltico; aperfeiçoamento de projetos. }\end{array}$ \\
\hline $\begin{array}{l}\text { Pinto, Santos e Catunda } \\
\text { (2015) }\end{array}$ & $\begin{array}{l}\text { Sistema de gestão de qualidade do processo construtivo a cargo da empresa } \\
\text { construtora para a redução de perdas por meio da implementação de práticas } \\
\text { sustentáveis que contribuem para a melhoria do desempenho ambiental e da } \\
\text { qualidade dos serviços executados. }\end{array}$ \\
\hline Wang, Li e Tam (2015) & Projetos de baixo desperdício com a escolha de materiais pré-moldados. \\
\hline Dondo (2017) & $\begin{array}{l}\text { Substituição dos materiais tradicionais por materiais alternativos, como: } \\
\text { estruturas metálicas e pré-moldadas e materiais recicláveis (agregados } \\
\text { reciclados de RCC). }\end{array}$ \\
\hline
\end{tabular}

\section{Fonte: Autores}

\section{Quadro 3 - Ações de Reutilização dos RCC}

\begin{tabular}{|l|l|}
\hline Autor/Ano & Ações de Reutilização \\
\hline Gangolells et al. (2014) & $\begin{array}{l}\text { Planejamento prévio da desmontagem seletiva de estruturas sobre a } \\
\text { demolição, para que seja possível a sua reutilização. }\end{array}$ \\
\hline Penteado e Rosado (2016) & Reutilização dos RCC como materiais de pavimentação. \\
\hline $\begin{array}{l}\text { Ribeiro, Moura e Pirote } \\
(2016)\end{array}$ & $\begin{array}{l}\text { Reutilização dos resíduos de cerâmicas em trabalhos artísticos como } \\
\text { mosaicos, em escadas ou em paredes. }\end{array}$ \\
\hline Tam e Hao (2016) & Reutilização dos resíduos de concreto para nivelar o canteiro de obras. \\
\hline Leite et al. (2018) & Reutilização dos resíduos de solos, areia e argila em obras viárias. \\
\hline $\begin{array}{l}\text { Santos e Marchesini } \\
(2018)\end{array}$ & $\begin{array}{l}\text { Reutilização de resíduos de solo para reaterros, de madeira para construções } \\
\text { provisórias e sinalizações, de metais para andaimes e proteções para altura. }\end{array}$ \\
\hline
\end{tabular}

Fonte: Autores

Quadro 4 - Ações de Reciclagem dos RCC

\begin{tabular}{|l|l|}
\hline Autor/Ano & Ações de Reciclagem \\
\hline Gangolells et al. (2014) & Reciclagem de resíduos inertes por meio da sua trituração. \\
\hline Bakshan et al. (2015) & Revenda dos resíduos de aço para empresas de reciclagem. \\
\hline Brasileiro e Matos (2015) & $\begin{array}{l}\text { Reciclagem de RCC para uso como camadas de base e sub-base para } \\
\text { pavimentação, coberturas primárias de vias, fabricação de argamassa; } \\
\text { reciclagem a partir do processo de moagem para uso na contenção de } \\
\text { encostas, calçamento e blocos de concreto; utilização dos resíduos de } \\
\text { cerâmica vermelha para a confecção de tijolos. }\end{array}$ \\
\hline Galarza et al. (2015) & $\begin{array}{l}\text { Transformação de RCC em agregados reciclados para a fabricação de blocos } \\
\text { de concreto não estruturais. }\end{array}$ \\
\hline Penteado e Rosado (2016) & $\begin{array}{l}\text { Transformação de RCC em agregados reciclados para serem utilizados na } \\
\text { construção de estradas. }\end{array}$ \\
\hline $\begin{array}{l}\text { Ribeiro, Moura e Pirote } \\
\text { (2016) }\end{array}$ & $\begin{array}{l}\text { Reciclagem do metal para utilização em telhas metálicas, sapatas e pré- } \\
\text { moldados. }\end{array}$ \\
\hline Tam e Hao (2016) & $\begin{array}{l}\text { Reciclagem por meio da moagem de resíduos de drywall para serem } \\
\text { utilizados como fertilizantes para solos. }\end{array}$ \\
\hline $\begin{array}{l}\text { Monteiro, Amaral } \\
\text { Delgado (2017) }\end{array}$ & $\begin{array}{l}\text { Transformação de RCC em agregados reciclados para confecção de concreto } \\
\text { não estrutural, como concreto magro, meio-fio e calçadas. }\end{array}$ \\
\hline Leite et al. (2018) & $\begin{array}{l}\text { Reciclagem de metais ferrosos para utilização em empresas de metalurgia; } \\
\text { reciclagem de resíduos de gesso para utilização na agricultura. }\end{array}$ \\
\hline $\begin{array}{l}\text { Alves e Santos (2019) } \\
\text { (2020) }\end{array}$ & $\begin{array}{l}\text { Utilização de restos de madeira para confecção de brinquetes que abastecem } \\
\text { um forno industrial. }\end{array}$ \\
\hline hotta e Bernucci & $\begin{array}{l}\text { Utilização de agregados reciclados junto ao cimento Portland e a cal } \\
\text { hidratada como base para pavimentação. }\end{array}$ \\
\hline
\end{tabular}


De acordo com os autores abordados nos Quadro 2, 3 e 4, há diversas práticas de redução, reutilização e reciclagem dos RCC dentro e fora dos canteiros de obras. No estudo de caso foram analisadas quais práticas são realizadas no canteiro.

\section{RESULTADOS OBTIDOS}

A realização do estudo de caso proporcionou o conhecimento de outras práticas relacionadas aos 3Rs. A coleta das informações foi realizada a partir da aplicação do checklist na obra e as imagens foram capturadas durante a visita ao canteiro.

Quadro 5 - Dados referentes ao estudo de caso

\begin{tabular}{|c|c|c|}
\hline Dados da empresa & Dados do respondente & Dados da obra \\
\hline $\begin{array}{l}\text { A empresa foi fundada em } \\
1983 \text { em Pernambuco. É } \\
\text { considerada uma empresa } \\
\text { de grande porte (mais de } \\
500 \text { funcionários). }\end{array}$ & $\begin{array}{l}\text { A respondente do checklist } \\
\text { é engenheira civil da } \\
\text { empresa e acompanha a } \\
\text { obra desde o seu início. }\end{array}$ & $\begin{array}{l}\text { A obra } 1 \text { teve início em março de } 2019 \text { e tem } \\
\text { previsão de término em maio de } 2022 \text {. Consiste } \\
\text { em um edifício residencial (conjunto de } \\
\text { apartamentos) composto por } 20 \text { pavimentos. A } \\
\text { obra contém } 7.630,85 \mathrm{~m}^{2} \text { de construção e } \\
\text { certificação ambiental ISO } 14001 \text {. }\end{array}$ \\
\hline
\end{tabular}

Fonte: Autores

No Quadro 5 são apresentados os dados da empresa, do respondente e da obra em que o checklist foi aplicado. Tal obra foi escolhida devido ao indicador de geração de resíduos da respectiva empresa construtora ser menor do que a média quando comparado com outras obras na cidade do Recife, estado de Pernambuco, de acordo com a pesquisa realizada na EMLURB por Lins (2020).

Quanto ao PGRCC, na obra, a elaboração e implantação é realizada pelo setor de engenharia civil em conjunto com o setor de engenharia ambiental da empresa. Além do monitoramento constante, é feita uma auditoria a cada 2 meses na obra, visando verificar se está sendo cumprido o que foi planejado. É realizado um treinamento com os funcionários para instruir a execução correta das etapas do PGRCC. Em relação a estas etapas, foi observado que a caracterização é realizada por meio da identificação e da quantificação de resíduos químicos, EPIs usados, plásticos, papel, madeira, e lixo não reciclável; a segregação é realizada por meio da separação dos resíduos em classes estabelecidas pela Resolução CONAMA n 307; o acondicionamento desses resíduos é realizado de acordo com o projeto de layout do canteiro e é feito em baias, caçambas estacionárias e tambores plásticos; o transporte dentro do canteiro é realizado principalmente de forma vertical, através do duto coletor de resíduos, apresentado na Figura 2, o qual facilita o transporte de resíduos de todos os pavimentos até a caçamba estacionária. Os resíduos do canteiro são retirados por uma empresa devidamente licenciada que recebe todos os tipos de RCC, inclusive gesso, metal, plástico, madeira e papel; a mesma dispõe de maquinários adequados para processamento e reciclagem dos resíduos, produzindo novos materiais. Com relação ao layout do canteiro de obras, o projeto de locação da obra já contém a locação dos armazenadores de resíduos.

Na obra investigada são adotadas as seguintes ações de redução e de reutilização dos RCC:

- Realização da compatibilização de projetos anteriormente ao início da obra por uma empresa responsável pela gestão de projetos;

- Realização de um estudo para o controle de produção de materiais no canteiro de obras. A engenheira civil, respondente do checklist, afirmou que esse estudo é feito a partir do índice hora/homem especificados em projetos e de históricos de obras anteriores, contribuindo para evitar eventuais desperdícios de materiais;

- Implantação da logística reversa com os paletes de materiais cerâmicos, os quais, após o uso, retornam para a fábrica em que foram adquiridos; 
- Execução de projeto de alvenaria racionalizada (Figura 3). Conforme afirmado pela respondente do checklist, um grande fator que aumenta a geração de resíduos na fase de execução da alvenaria consiste em quebras de parte dos blocos cerâmicos para a passagem de instalações. Devido a isso, a adoção de projeto de alvenaria racionalizada com furos verticais para as passagens é essencial para a redução de resíduos cerâmicos;

- Utilização de argamassa estabilizada. Tal argamassa é dosada com aditivo retardador de pega que prolonga seu uso até 24 horas após a adição de água. A engenheira civil afirmou que é perceptível uma redução de resíduos de argamassa gerados em relação a argamassa convencional que, ao contrário da estabilizada, possui tempo de utilização de 2 a $3 \mathrm{~h}$, aumentando o descarte do que não foi utilizado dentro desse período. Ainda segundo ela, como a argamassa estabilizada possui o período de uso mais longo, possibilita a utilização de praticamente toda a argamassa comprada, havendo um mínimo descarte;

- Execução de passagens pré-definidas durante a etapa de concretagem para realização de instalações diversas. Segundo a engenharia civil, as passagens para tubulações elétricas e hidráulicas são feitas na etapa da concretagem de vigas e pilares. De acordo com ela, o objetivo é evitar a realização de furos após a concretagem, os quais, caso fossem realizados, gerariam resíduos de concreto. Por isso, evitar a execução deles é uma forma de redução da geração de resíduos.

- Reutilização de resíduos de solo para reaterros dentro do próprio canteiro.

Figura 2 - Duto coletor fazendo o transporte de resíduos até a caçamba estacionária

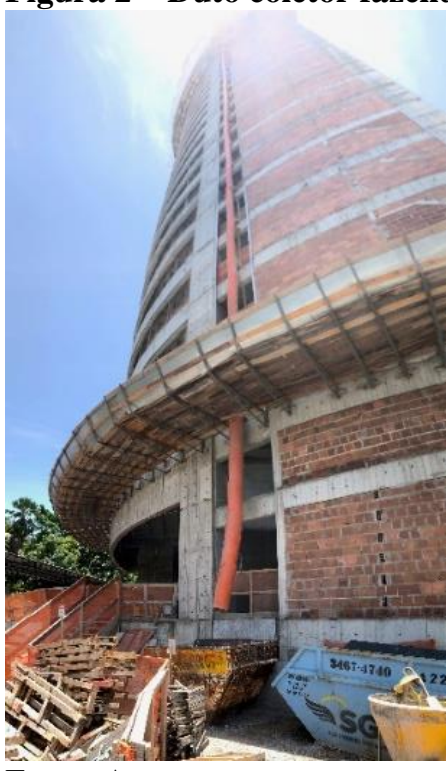

Fonte: Autores

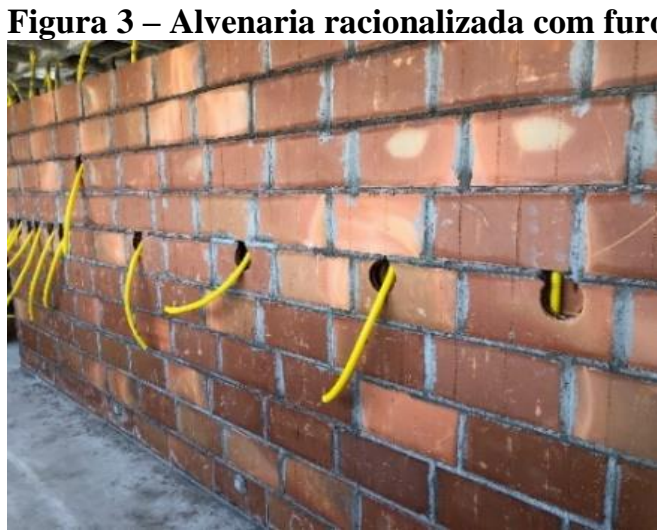

Fonte: Autores 


\section{CONSIDERAÇÕES FINAIS}

Conclui-se, através dos resultados obtidos na revisão bibliográfica e nos estudos de casos, que é possível melhorar a gestão dos resíduos da construção civil, nos canteiros de obras, através da adoção de práticas de redução, reutilização e reciclagem que auxiliem no tratamento ambientalmente adequado dos RCC.

Foi possível identificar, como importantes contribuições para a redução de resíduos da construção civil na obra investigada, as seguintes ações: compatibilização de projetos, estudo para o controle de produção de materiais no canteiro de obras, implantação da logística reversa, execução de projeto de alvenaria racionalizada, utilização de argamassa estabilizada, e execução de passagens pré-definidas durante a etapa de concretagem para realização de instalações diversas. A ação de reutilização de resíduos identificada foi o uso de resíduos de solo para reaterros na própria obra.

Dessa maneira, a sistematização de ações de redução, reutilização e reciclagem que foi realizada nesta pesquisa por meio da revisão bibliográfica e da pesquisa de campo, contribui para a identificação das boas práticas implantadas. Essas práticas podem ser replicáveis para a melhoria dos canteiros de obras, no que se diz respeito a gestão de resíduos da construção civil.

\section{REFERÊNCIAS}

ALVES, T. E. R.; SANTOS, M. S. F. A gestão de resíduos da construção civil em Teresina - Piauí. REEC-Revista Eletrônica de Engenharia Civil, v. 15, n. 1, 2019.

Associação Brasileira para Reciclagem de Resíduos da Construção Civil e Demolição ABRECON. Relatório da pesquisa setorial 2017/2018.

Associação Brasileira para Reciclagem de Resíduos da Construção Civil e Demolição ABRECON. Relatório da pesquisa setorial 2014/2015. ABRECON/UFPR. São Paulo, 2015. 30p.

BAKSHAN, A. et al. A field based methodology for estimating waste generation rates at various stages of construction projects. Resources, Conservation and Recycling, v. 100, p. 70-80, 2015.

BEJA, I. A.; MOTTA, R.; BERNUCCI, L. B. Application of recycled aggregates from construction and demolition waste with Portland cement and hydrated lime as pavement subbase in Brazil. Construction and Building Materials, v. 258, p. 119520, 2020.

BRASIL. Resolução CONAMA n 307, de 05 de julho de 2002. Estabelece diretrizes, critérios e procedimentos para a gestão dos resíduos da construção civil. Diário Oficial da República Federativa do Brasil, 2002.

BRASILEIRO, L. L.; MATOS, J. M. E. Revisão bibliográfica: reutilização de resíduos da construção e demolição na indústria da construção civil. Cerâmica, v. 61, n. 358, p. 178-189, 2015.

DONDO, M. V. M. Avaliação da gestão de resíduos da construção civil em Cuiabá e Várzea Grande. Revista DAE, 2017.

GALARZA, L. H. W. et al. Modelo dinâmico de sistemas para o gerenciamento de resíduos da construção civil na cidade de Porto Alegre: estudo de caso. Engenharia Sanitaria e Ambiental, v. 20, n. 3, p. 463-474, 2015.

GANGOLELLS, M. et al. Analysis of the implementation of effective waste management practices in construction projects and sites. Resources, conservation and recycling, v. 93, p. 99-111, 2014.

GIL, A. C. Como elaborar projetos de pesquisa. 4 ed. Atlas. São Paulo, 2010. 
LEITE, I. C. A.; DAMASCENO, J. L.; REIS, A. M.; ALVIM, M. Gestão de resíduos na construção civil: um estudo em belo horizonte e região metropolitana. REEC - Revista Eletrônica de Engenharia Civil, v. 14, n. 1, p. 159, 2018.

LINS, E. J. M. Proposta de indicadores de resíduos de construção e demolição (RCD) de edificações na cidade do Recife. Dissertação (Mestrado). Escola Politécnica de Pernambuco, Universidade de Pernambuco, p. 240, 2020.

MONTEIRO, R. R.; AMARAL, D.; DELGADO, S. Utilização de resíduos provenientes da construção e demolição de reforma residencial como agregado miúdo para fabricação de concreto. Revista Teccen, 2017.

PENTEADO, C. S. G.; ROSADO, L. P. Comparison of scenarios for the integrated management of construction and demolition waste by life cycle assessment: A case study in Brazil. Waste management \& research, v. 34, n. 10, p. 1026-1035, 2016.

PINTO, C. H. C.; SANTOS, A. L.; CATUNDA, A. C. M. M. Percepção da legislação ambiental, gestão e destinação final dos RCD - Resíduos da Construção e Demolição: um estudo de caso em Parnamirim/RN/Brasil. HOLOS, v. 2, p. 33-49, 2015.

RIBEIRO, D.; DE MOURA, L. S.; SANTOS PIROTE, N. S. Sustentabilidade: formas de reaproveitar os resíduos da construção civil. Revista de Ciências Gerenciais, v. 20, n. 31, p. 41-45, 2016.

SANTOS, M. H. S.; MARCHESINI, M. M. P. Logística reversa para a destinação ambientalmente sustentável dos resíduos de construção e demolição (RCD). Revista Metropolitana de Sustentabilidade (ISSN 2318-3233), v. 8, n. 2, p. 67-85, 2018.

SILVA, W. C.; SANTOS, G. O.; ARAÚJO, W. E. L. Resíduos Sólidos de Construção Civil: caracterização, alternativas de reuso e retorno econômico. Revista Gestão \& Sustentabilidade Ambiental, v. 6, n. 2, p. 286-301, 2017.

TAM, V. WY; HAO, J. JL. Attitudes towards recycling on construction sites. In: Proceedings of the Institution of Civil Engineers-Waste and Resource Management. Thomas Telford Ltd, 2016. p. 131-136.

WANG, J.; LI, Z.; TAM, V. W. Y. Identifying best design strategies for construction waste minimization. Journal of cleaner production, v. 92, p. 237-247, 2015. 